\title{
MODELAMIENTO MATEMÁTICO COMO HERRAMIENTA DE ARTICULACIÓN DE LA MATEMÁTICA UNIVERSITARIA EN ESTUDIANTES DE PRE CÁLCULO
}

\author{
Mathematical modeling as a tool of interaction \\ of mathematics university students of precalculus
}

JUAN GUILlERMO NÚÑEZ OSUNA*, ALFONSO SÁNCHEZ BERNAL**

Recibido: 4 de Noviembre de 2015. Aceptado: 22 de Noviembre de 2015

DOI: http://dx.doi.org/10.21017/rimci.2016.v3.n5.a4

\begin{abstract}
Resumen
En la investigación se indaga sobre el rendimiento de los estudiantes del curso de pensamiento matemático a partir del cual se diseña una estratégica didáctica apoyada en componentes tales como la educación matemática, pensamiento variacional, cerebro tríadico y competencias como sustento teórico con el propósito de fortalecer el concepto de función para posteriores cursos de cálculo. El objetivo es desarrollar una estrategia didáctica centrada en el modelamiento matemático para el afianzamiento del concepto de función en los estudiantes del curso de pensamiento matemático de la jornada nocturna de la Universidad Jorge Tadeo Lozano. El propósito de esta investigación es el de establecer el rendimiento académico de los estudiantes de un curso de pensamiento matemático para diseñar una estratégica didáctica que apoyada en la tricerebral y el modelamiento permita la consolidación del concepto de función. El método fue desde el enfoque mixto mediante la aplicaccion el instrumento, se recogio la informacion en forma de prueba diagnostica, revision de notas, talleres y pruebas para ofrecer una visión conjunta, esta continua con el diseño de la propuesta. Como conclusión encontramos que desde nuestra propuesta se hace posible consolidar de manera integral el metalenguaje, la competencia numérica y el pensamiento variacional en lo referente con el concepto de función.
\end{abstract}

Palabras clave: cerebro tríadico, competencias matemáticas, educación matemática, función, modelamiento matemático, pensamiento variacional.

\begin{abstract}
In the research it is investigated about the performance of students in the course of mathematical thought from which is designed a teaching strategy supported by components such as mathematics education, variational thinking, triadic brain and skills as theoretical support in order to strengthen designing the function concept for future courses of calculus. The objective is develop an educational strategy focused on mathematical modeling for the strengthening the concept of function in the students of mathematical thinking course, in the night shift at the Jorge Tadeo University. The main purpose of this research is to establish the academic performance of students in a course of mathematical thinking to design a didactic strategy supported in the tricerebral and this modeling allows the consolidation of function concept. The method
\end{abstract}

* Magíster en Educación de la Universidad Cooperativa de Colombia. Especialista en docencia universitaria de la Universidad Cooperativa de Colombia y Licenciado en matemáticas y física de la Universidad de los Llanos. Diplomados en tutor virtual de curso académico. E-Mediador y diseñador en AVA de la UNAD. Diplomado en Gerencia Educativa en el Politécnico Superior de Colombia. Docente de Ciencias Básicas Corporación Unificada Nacional Superior. Docente de especializaciones, Universidad Nacional Abierta y A Distancia UNAD. Correo electrónico: juannuosuna@gmail.com

** Magíster en Educación de la Universidad Cooperativa de Colombia, Especialista en Docencia Universitaria de la Universidad Cooperativa de Colombia y Licenciado en Matemáticas de la Universidad Pedagógica Nacional. Docente de Ciencias Básicas en la Universidad Jorge Tadeo Lozano. Correo electrónico: alfsancez21@hotmail.com 
was conceived from the mixed approach through the application of the instrument, the information was collected as diagnostic test, notes review, workshops and testing to offer a joint vision and this continues with the proposal design. In conclusion, we could find that from our proposal is possible to consolidate of integral manner the metalanguage, the numerical competency and variational thought, in regard to the concept of function.

Keywords: triadic brain, math skills, mathematics education, function, mathematical modeling, variational thinking.

\section{INTRODUCCIÓN}

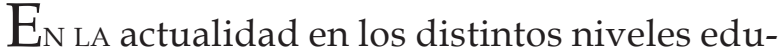
cativos encontramos diversas dificultades en la comprensión, aplicación y apropiación del concepto de función. En muchos casos los estudiantes asocian algunas características de la función, pero, finalmente llegan a la universidad entendiendo este concepto de forma errónea o bastante sesgada y limitada. Esta problemática se evidencia en los cursos regulares de precalculo, el cual se centra en el estudio de dicha temática; la intención de este trabajo, por consiguiente, es realizar un análisis acerca de las dificultades que presentan los estudiantes del curso regular de precalculo en cuanto a tareas de interpretación y representación asociadas al concepto de función y proponer una alternativa de enseñanza de dicho concepto utilizando como herramienta la modelación matemática.

Por medio de las funciones se pueden abordar diversas situaciones cotidianas, de igual forma, observamos que está presente en gran variedad de fenómenos en distintos campos del conocimiento, casi todo a nuestro alrededor es susceptible de ser tratado a través del planteamiento y estudio de una o varias funciones.

Al respecto señala Spivak: «El concepto más importante de todas las matemáticas es, sin dudarlo, el de función: en casi todas las ramas de la matemática moderna, la investigación se centra en el estudio de funciones»[1].

Una de las metas que se debe proponer la educación matemática es la de desarrollar en los estudiantes las competencias necesarias para entender que el cambio está y ha estado presente en todos los estamentos sociales, económicos y políticos, que son la base de nuestra sociedad; al respecto señala Stewart: «Es de la mayor importancia la necesidad de entender y controlar el mundo cambiante en que vivimos»[2].
Consideramos que para lograr que los estudiantes realmente consigan apropiarse del concepto de función, y de cualquier otro, es fundamental dejar atrás las prácticas y clases rutinarias, es necesario cambiar la metodología tradicional basada en el cerebro izquierdo, en donde solo se privilegia el conocimiento e implementar una nueva estrategia para la enseñanza de las matemáticas, en este caso la enseñanza del concepto de función.

La modelación matemática se presenta Como una herramienta poderosa para tratar la problemática anteriormente descrita, pues su utilización adecuada y efectiva sirve de puente para comprender y aplicar de manera significativa muchos conceptos y fenómenos de las ciencias exactas. Blum y Niss [3] proponen cinco argumentos, para la inclusión de la modelación en la enseñanza de las matemáticas:

\section{A. Formativo}

La modelación provee el conocimiento adecuado para desarrollar competencias generales y capacidades globales y creativas para la resolución de problemas.

\section{B. Competencia crítica}

Prepara a los estudiantes para vivir y actuar de forma íntegra, como ciudadanos críticos, en una sociedad cada vez más influenciada por las matemáticas.

\section{Utilidad}

Prepara a los estudiantes para resolver problemas y para describir aspectos de situaciones extramatemáticas.

\section{Imagen de las matemáticas}

Ofrece a los alumnos una imagen rica y comprensiva de todas las facetas de las matemáticas, Como ciencia, y como campo de actividad en la sociedad y en la cultura.

Rev. Ingeniería, Matemáticas y Ciencias de la Información Vol. 3 / Núm. 5 / enero-junio de 2016; pág. 37-50 


\section{E. Aprendizaje de las matemáticas}

Ayuda a los estudiantes en la adquisición y aprendizaje de conceptos matemáticos, nociones, métodos y resultados.

Las actividades que se presentan en la propuesta pretenden apuntar hacia estos argumentos y convertirse en un medio para que se potencie en los estudiantes destrezas y habilidades propias del pensamiento matemático a través de acciones de tipo lógico-analíticas, intuitivo-sintéticas y motoras-operacionales, es decir, prácticas que desarrollen la inteligencia racional, emocional y operacional, esto les va a proporcionar una manera natural para actuar en su entorno, de tal forma que estén en capacidad de razonar en forma coherente, desarrollando sensibilidad y creatividad, con la firme idea de mejorar constantemente.

Es importante destacar algunos estudios que sirvieron como soporte para la investigación, entre otros, encontramos a Carlos Guevara, quien establece como premisa el aprendizaje significativo para estudiar el concepto de función y desarrolla actividades de simulación y modelación, generando así procesos de meta cognición en los estudiantes [4].

De igual forma, Planchard, quien hace un análisis riguroso de la importancia de la modelación, la visualización y los sistemas de representación en el proceso didáctico para la adquisición del concepto de función y como la práctica docente se encasilla en esquemas convencionales de transmisión de conocimientos [5].

También, se resalta el trabajo de Franciscio Córdoba, quien plantea la importancia de la modelación en la enseñanza de las matemáticas, pues es el medio que permite a los estudiantes el desarrollo de procesos de resignificacion que dan dinamismo al conocimiento matemático, ya no aislado y desvinculado de la realidad [6].

\section{FundAMENTACIÓN TEÓRICA}

En relación con el marco teórico, se ha considerado reconocer aspectos tales como educación matemática, didáctica de las matemáticas, competencias matemáticas y cerebro tríadico.

\section{A. Función}

El concepto de función es, tal vez, una de las temáticas más importantes a lo largo de la historia en el desarrollo de las matemáticas, puesto que, se ha utilizado con gran eficacia en distintas ramas del conocimiento, en efecto, permite modelar o describir y por consiguiente analizar gran cantidad de fenómenos de diversa índole.

Para entender el concepto de función afirma William Ugalde, se deben tener en cuenta tres hechos importantes, ellos son [7]:

1) Que el concepto de función está intimamente ligado al concepto primitivo de conjunto (al igual que de otros conceptos como relación, variable, criterio, etc.).

2) Que el concepto de función desde su origen cualquiera que este sea-, está ligado al desarrollo del concepto de cantidad, y más generalmente, al concepto de número.

\section{3) Que el concepto de función nace del interés de la} humanidad por entender el mundo que le rodea.

Para descubrir el nacimiento de este concepto debemos remitirnos a la antigüedad, desde la época de los babilonios ya se trabajaban tablas con los cuadrados, los cubos y los inversos de los números naturales. Se puede afirmar que estas tablas definen funciones de $\mathrm{N}$ en $\mathrm{N}$ o de $\mathrm{N}$ en $\mathrm{R}$.

En Grecia, figuran trabajos sobre proporción, a través de la relación entre magnitudes de igual tamaño, se distinguen relaciones entre magnitudes dependientes. En la edad media, se presentan estudios sobre el cambio, en particular al movimiento, aparecen conceptos fundamentales como la cantidad variable y de ésta forma se avanza en la idea de relación funcional. Esto se podría catalogar como las actuales variables dependiente e independiente, como lo afirma Crombie, citado por Ruiz [8].

Isaac Newton se dedicó a reestructurar las bases del cálculo, encontrando que fuxión es la velocidad con que la variable se transforma con el tiempo. Jean Bernoulli afirmo que una función es una cantidad compuesta de tal manera que dentro de ella encontramos variable y constantes. Leonard Euler realiza un estudio sistemático de todas las funciones, define las funciones continuas 
y finalmente gracias a él tenemos la actual notación de función $\mathrm{f}(\mathrm{x})$. Joseph Lagrange interpreto la función desde la perspectiva analítica fundamental para el desarrollo de los fundamentos del análisis, la elaboración de teoría de funciones. Joseph Baptiste Fourier representa la función en términos de sucesiones de valores y ordenadas las cuales están sujetas a una regla común.

Gustave Dirichlet estudio el concepto de función Como una variable independiente en un intervalo determinado, el concepto de dominio y rango de una función. Formula el concepto moderno «y es una función de una variable $x$, definida en el intervalo $a<x<b$, si a todo valor de la variable $x$ en este intervalo le corresponde un valor definido de la variable y» según Kleiner, referido por Carlos Cuevas y Jose Díaz en «La historia de la matemática un factor imprescindible en la elaboración de una propuesta didáctica» [9].

En 1939 el grupo Bourbaki caracteriza una relación de correspondencia entre un dominio y rango, donde ambos conjuntos son arbitrarios «Sean $E$ y $F$ dos conjuntos, que pueden ser distintos. Una relación entre un elemento variable $x$ de $E y$ un elemento variable y de F se llama una relación funcional en $y$, si para toda «x», existe un único «y» en «F» el cual está en la relación dada con $x »$, según Rüting, nuevamente referido por Carlos Cuevas y Jose Díaz en «La historia de la matemática un factor imprescindible en la elaboración de una propuesta didáctica [9].

Existen diferentes formas de representar una función:

\section{a) Descripción verbal}

Es un enunciado en el que se describe el comportamiento de fenómenos naturales que implica una relación entre dos o más variables.

\section{b) Tablas}

Corresponden a un listado organizado en dos filas o columnas de valores de la variable independiente y los correspondientes a variable.

Tabla 1. Listado Organizado.

\begin{tabular}{lccccc}
\hline Unidades & 1 & 2 & 3 & 4 & 5 \\
Costo total & 20 & 25 & 30 & 35 & 40 \\
\hline
\end{tabular}

Fuente: Los autores.

\section{4) Gráficas}

Es la representación en el plano mediante una línea recta, curva u otro tipo de forma evidenciando una relación entre variables.

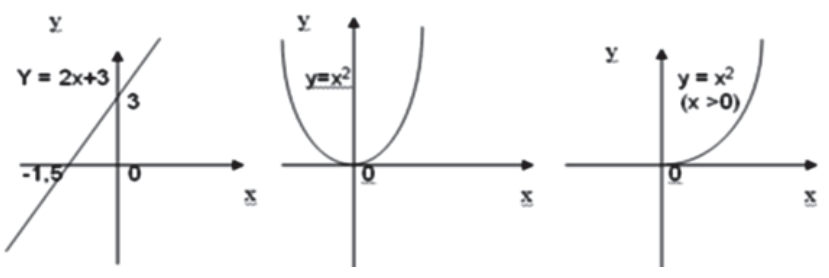

Fig. 1. Representación en el plano.

\section{5) Expresiones algebraicas}

Son fórmulas que relacionan las dos variables que intervienen en una función, correspondiente a lectura de relaciones simbólicas.

$$
\begin{aligned}
& f(x)=x^{2}-3 x+2 \\
& y(t)=v t-\frac{1}{2} \quad g t^{2}
\end{aligned}
$$

En conclusión las representaciones son un instrumento muy importante para la comprensión de los procesos de enseñanza y aprendizaje en matemáticas además de las tareas de interpretación y argumentaciones de muchas situaciones matemáticas las cuales permiten la adquisición y consolidación del concepto de función y sus diferentes usos. De otra parte es importante mencionar que los tipos de representaciones han evolucionado a medida que cambian y se trasforman las diferentes sociedades en cada una de las épocas.

\section{EduCACión MATEMÁTICA}

La Matemática Educativa está relacionado con aquellos factores que intervienen y hacen posible que la Matemática se enseñe y se aprenda, por lo que en la última década se ha reconocido por diversos autores la influencia determinante que sobre ella ejercen las posiciones filosóficas, las teorías epistemológicas relativas al conocimiento matemático desde diferentes contextos. Seguidamente encontramos que gracias al sentido constructivista de ella todas las actividades que se desarrollan corresponden a procesos cuyos resultados se

Rev. Ingeniería, Matemáticas y Ciencias de la Información Vol. 3 / Núm. 5 / enero-junio de 2016; pág. 37-50 
enmarcan en los contextos sociales, culturales e históricos que se realizan como resultado el trabajo tanto individual y colectivo en coherencia con los objetos e investigaciones matemáticas que adelantan actualmente.

De otra parte según lo expuesto por A. Robert y Royalsk referido por Artigue, el doble enfoque de la enseñanza de las matemáticas se reconoce en el ergonómico cognitivo y la didáctica de las matemáticas donde el docente realiza su labor en un ambiente dinámico y abierto, particularmente difícil y exigente de competencias [10]. Desde esta perspectiva encontramos nuevos retos y tareas a desarrollar en nuestra labor docente, que necesita y requiere una serie actividades apoyadas en las prácticas, modos de evolución del oficio docente.

El estudio de la didáctica de la matemática y la educación matemática incorpora herramientas materiales y simbólicas asociadas a las matemáticas como ciencia, de otra parte la evolución de los enfoques teóricos hacia los paradigmas de la naturaleza sociocultural y antropológica están asociados a la evolución tecnológica generando un nuevo contexto en las prácticas educativas en los diferentes niveles de escolaridad [10].

Es así como aprender matemáticas se ha convertido en la elaboración de definiciones, buscar ejemplos y aplicaciones para encontrar sentido a los teoremas y otros elementos del objeto matemático desde la aplicabilidad y la existencia en la realidad objetiva, al respecto Miguel de Guzmán quién a partir del análisis de los principales movimientos, transformaciones y resultados en las últimas décadas concluye que el objeto de la actividad matemática se manifiesta en modos peculiares de tratamiento que incluyen: una simbolización adecuada, una manipulación racional rigurosa y un dominio efectivo de la realidad a la que se dirige [10].

Es así como la matemática educativa en general se refiere al estudio de los fenómenos didácticos relacionados con el saber matemático desde la que se generan problemas teóricos y prácticos referidos a la adaptación del saber matemático y del saber científico con la finalidad de usar este resultado en la mejora de los procesos educativos. Por tanto en ella confluye la naturaleza epistemológica, planos cognitivos y transmisión de conocimiento, con referencia a nuestra propuesta incorporamos los elementos propuestos por Tall y Vinner nos establecen lo siguiente: «La relación conceptual de los estudiantes se encuentra referida a la experiencia y la estructura cognitiva asociada al concepto» [10].

\section{Dificultades}

A lo largo de la historia encontramos diferentes conceptos de función tales que como la relación entre variables, una función numérica como la aplicación de un conjunto $\mathrm{E}$ y una relación de $\mathrm{X}$ e U. La función relación entre conjuntos de números, tal que sea la existencia de un dominio con respecto al rango y en nuestro caso no es diferente.

La función es una expresión matemática que puede ser representada, en términos de rectas, curvas continuas, y redondas y parábolas entre otras. Generalmente los estudiantes forman un concepto de función como resultado de diferentes funciones estudiadas, adicionalmente a ello se debe a que estas se presentan en términos de fórmulas, la lectura común de libros y la enseñanza llega a desarrollar modelos diferentes del contenido formal general una errada trasposición didáctica.

Los conceptos dominio y rango son fuente de bastantes errores por parte de los estudiantes en el campo de las matemáticas en los cursos de pre calculo y en nuestro caso es un curso de pensamiento matemático.

Dada la importancia de esta temática y su influencia en la enseñanza de matemáticas y en cursos posteriores hemos creado las siguientes categorías:

\section{A. Proceso de aprendizaje}

Dependencia entre variables, pero no se comprende la regla que domina dicha relación, muchos de los estudiantes no son capaces establecer la relación entre las variables de una función en nuestro estudio encontramos lo mismo [11]. Otros estudiantes logran definir la función, pero fracasan [12], es decir si una gráfica representa o no 
una función, en tal caso tendremos lo expuesto por Tall y Vinner [13]:

La existencia de una dependencia entre dos variables, cuyo significado no se comprende e influye en la respuesta del estudiante cuando debe clasificar que relaciones son funciones y cuáles no.

Las funciones y ejemplos de funciones estudiadas en clase, como recuerdos inmediatos o prototipos, que necesita el estudiante para responder una pregunta o para abordar la solución de un problema.

De acuerdo con lo anterior, tenemos que:

«La estructura cognitiva de nuestros estudiantes de la función es creciente, decreciente y existencia de extremos, en particular cuando ella se visualizan mediante la representación gráfica» [14].

\section{B. Las tareas de construcción como formas de pre- sentar una función}

Afectadas cuando se desarrollan en el contexto geométrico dado la importancia y valor que tiene las gráficas en este proceso tendremos lo siguiente [15]:

La incomprensión entre el lenguaje común crea un conflicto en la tarea a concebir la función desde lo geométrico.

El nivel de conocimiento sobre el contexto geométrico para construir diferentes formas de representación del concepto de función, con lo cual no es posible tener una definición integral del concepto de función.

Muchos estudiantes no pueden transferir la función, de la tabla a la gráfica o viceversa, por tanto no considerar el proceso integral.

Como conclusión para este caso muchos de los estudiantes no pueden trasladar del lenguaje geométrico al lenguaje de funciones [16] tal que la habilidad para establecer relación entre los diferentes sistemas de representación y la dificultad para la construcción, pues consiste en pasar de la descripción verbal o grafica o tabla [15].

\section{La comprensión del lenguaje común en el proceso de aprendizaje de los contenidos y conceptos}

Con respecto a esta dificultad, el conocimiento resulta determinante en el proceso de aprendizaje. La dificultad se halla en que el estudiante no posee la representación adecuada, los conceptos que intenta estudiar, provocando desconexión entre el sistema lingüístico y el contenido matemático [10][15].

\section{COMPETENCIAS}

El término competencia nace en el sector industrial para designar el conjunto de elementos y factores indispensables para alcanzar el éxito en el desempeño profesional. El sector educativo hace ya algún tiempo tomo este concepto y lo ha ido transformando, sin embargo, el significado de competencia y formación por competencias no resulta ser muy diferente al usado en el sector productivo; «la competencia se puede expresar como la capacidad para realizar una tarea, haciéndolo de manera eficaz; midiendo esta eficacia a través de resultados buscados vs logrados, la calidad del proceso seguido y el grado de fiabilidad de la solución al problema» [17].

Según el Ministerio de Educación Nacional, competencia es un conjunto de conocimientos, habilidades, actitudes, comprensiones, y disposiciones cognitivas, socio afectivas y sicomotoras, apropiadamente relacionadas entre sí para facilitar el desempeño flexible, eficaz y con sentido de una actividad o de cierto tipo de tareas en contextos nuevos y retadores [18].

Se trata, por tanto, de un «saber hacer», un saber que se aplica y es susceptible de adecuarse a una diversidad de situaciones y contextos y tiene un carácter integrador, abarcando conocimientos, procedimientos y actitudes [19].

En síntesis, ser competente es: interpretar de la mejor manera un determinado problema, saber con exactitud que recursos necesita para resolverlo y encontrar alternativas de solución, dando respuestas adecuadas e innovadoras que favorezcan su entorno.

Rev. Ingeniería, Matemáticas y Ciencias de la Información Vol. 3 / Núm. 5 / enero-junio de 2016; pág. 37-50 
Uno de los fines de la educación matemática es el de propender porque en la sociedad actual, cada vez más influenciada por esta disciplina, todas las personas tengan la posibilidad de interpretar críticamente la gran cantidad de información proveniente de diferentes fuentes, desenvolverse de manera natural en su entorno y tengan la claridad y seguridad necesaria a la hora de tomar decisiones ante cualquier suceso.

PISA define la competencia matemática como: «La capacidad del individuo para formular, emplear e interpretar las matemáticas en distintos contextos. Incluye el razonamiento matemático y la utilización de conceptos, procedimientos, datos y herramientas matemáticas para describir, explicar y predecir fenómenos [20]. Ayuda a los individuos a reconocer el papel que las matemáticas desempeñan en el mundo y emitir los juicios y las decisiones bien fundadas que los ciudadanos constructivos, comprometidos y reflexivos necesitan».

Se pueden establecer cuatro niveles de desarrollo de las competencias [21]:

Nivel 0: en este nivel se encuentran aquellas respuestas en las que no hay intento de resolver el problema, se dan explicaciones confusas que ponen de manifiesto que no hay comprensión alguna de la situación, o se establece relaciones erróneas entre las variables.

Nivel 1: identifica algunos aspectos relevantes de la situación pero sin comprenderla estructuralmente.

Nivel 2: identifica aspectos relevantes de la situación y establece sus relaciones mostrando comprensión estructural de la misma. Construye un modelo eficaz para abordar la búsqueda de respuestas sin un uso conveniente del mismo.

Nivel 3: construye un modelo eficaz que refleje el sentido dado por la situación y usa este modelo para tomar decisiones usándolo de manera adecuada.

Nivel 4: Comprueba la validez de las decisiones tomadas y procede, satisfactoriamente, a su comunicación.
En los estándares básicos de competencias se enuncian algunos procesos generales presentes en toda actividad matemática que pretenden explicar que es ser matemáticamente competente, ellos son: Formular y resolver problemas; modelar procesos y fenómenos de la realidad; Comunicar y utilizar diferentes registros de representación o sistemas de notación simbólica; razonar y usar la argumentación, la prueba y la refutación, el ejemplo y el contraejemplo; formular, comparar y ejercitar procedimientos y algoritmos.

En las pruebas saber $11^{\circ}$ se definen tres competencias que integran y recogen los elementos de estos procesos:

Interpretación y representación: Consiste en la habilidad para comprender y transformar la información presentada en distintos formatos como tablas, gráficos, conjuntos de datos, diagramas, esquemas, etcétera, así como la capacidad de utilizar estos tipos de representación para extraer de ellos información relevante que permita, entre otras cosas, establecer relaciones matemáticas e identificar tendencias y patrones.

Formulación y ejecución: La capacidad para plantear y diseñar estrategias que permitan solucionar problemas provenientes de diversos contextos, bien sean netamente matemáticos o del tipo de aquellos que pueden surgir en la vida cotidiana y son susceptibles de un tratamiento matemático. Se relaciona también con la habilidad o destreza para seleccionar y verificar la pertinencia de soluciones propuestas a problemas determinados, y analizar desde diferentes ángulos estrategias de solución.

Argumentación: Esta competencia se relaciona con la capacidad para validar o refutar conclusiones, estrategias, soluciones, interpretaciones y representaciones en situaciones problemáticas, dando razones del porqué, o del cómo se llegó a estas, utilizando ejemplos y contraejemplos, o bien señalando y reflexionando sobre inconsistencias presentes.

En los Lineamientos para la formación por competencias en educación superior, elaborados en 2010, los desempeños que se supone debe demostrar el estudiante son: 
Interpreta información presentada en gráficas, tablas, esquemas y problemas y a partir de ella, hace inferencias utilizando cálculos cuantitativos. Representa la información cuantitativa de diversas formas y utiliza métodos cuantitativos para solucionar problemas. Analiza, modela y elabora diferentes representaciones de una situación problema. Identifica alternativas de solución y sustenta su selección con criterio profesional. Evalúa la solución dada a un problema, las estrategias utilizadas y el impacto de su implementación en un contexto determinado.

\section{A. Modelación}

Un modelo puede entenderse como un sistema figurativo mental, gráfico o tridimensional que reproduce o representa la realidad en forma esquemática para hacerla más comprensible, Es una construcción o artefacto material o mental, un sistema que puede usarse como referencia para lo que se trata de comprender; una imagen analógica que permite volver cercana y concreta una idea o un concepto para su apropiación y manejo [18]

Sistema físico que sirve para representar un objeto, situación o fenómeno del mundo real, en el cual se utilizan relaciones y conceptos matemáticos, cuya utilidad está dada al ser una herramienta para interpretar, transformar y predecir dicho fenómeno [6].

La modelación permite entender un sinnúmero de situaciones y abordar problemas en diferentes áreas del conocimiento, es de utilidad para un sociólogo, ya que le permite conocer las variables que determinan el problema de la explosión demográfica; para un economista porque puede reconocer las variables que determinan la inflación, los factores que intervienen en la distribución del ingreso, la acumulación de capital o la evolución de los valores bursátiles, para un ingeniero, pues le permite prever la cantidad de energía que se requiere para una población en constante aumento, para un biólogo le ayudaría a identificar las variables que determinan el crecimiento de una población; en general lo podemos utilizar en todo tipo de fenómenos físicos, químicos o naturales, como la variación de la presión atmosférica, la velocidad y la aceleración, la gravitación universal, las leyes del movimiento, la función de onda de una partícula a escala cuántica y la desintegración de sustancias radiactivas.
Existen varios esquemas para representar el proceso de modelación matemática, la mayoría de ellos consta de cuatro etapas, a continuación se presenta el modelo elaborado por Blum, el cual nos parece pertinente por cuanto tiene en cuenta, no solamente las etapas presentes en este ciclo, sino que además, explicita los procesos que intervienen en cada una de las fases.

La Fig. 2 muestra el proceso de modelación propuesto por Blum, allí se describe paso a paso como resolver un problema, se identifican cuatro etapas:

1) Situación real o un problema dado en un contexto, en la cual se requiere obtener una meta, respuesta o decisión acerca de cómo se deberá proceder.

2) La generación de un modelo real, que permite identificar e interpretar el problema, además, reconocer las posibles variables.

3) La formulación de la situación-problema del mundo real en términos matemáticos, es decir, la construcción del modelo matemático, generalmente a través de ecuaciones o relaciones entre variables que describen la situación dada.

4) La solución y análisis del problema en términos matemáticos.

Este modelo se presenta como un ciclo, pues, una vez se llega a la última etapa, los resultados matemáticos se deben interpretar en el contexto de la situación real original, se debe verificar si el resultado obtenido corresponde con el problema planteado y efectuar un proceso de validación; en términos generales, se necesita adecuar la respuesta a la situación, si esto no es posible, se empezara de nuevo el ciclo, generar un nuevo modelo real y un nuevo modelo matemático, hasta que la respuesta obtenida pueda satisfacer las condiciones del problema.

\section{Cerebro}

Tricerebrar significa que hay que usar los tres cerebros o los tres procesos-información creativa creatividad, acción-siempre integrados, formando un ciclo que solo se completa cuando se cumplen

Rev. Ingeniería, Matemáticas y Ciencias de la Información Vol. 3 / Núm. 5 / enero-junio de 2016; pág. 37-50 


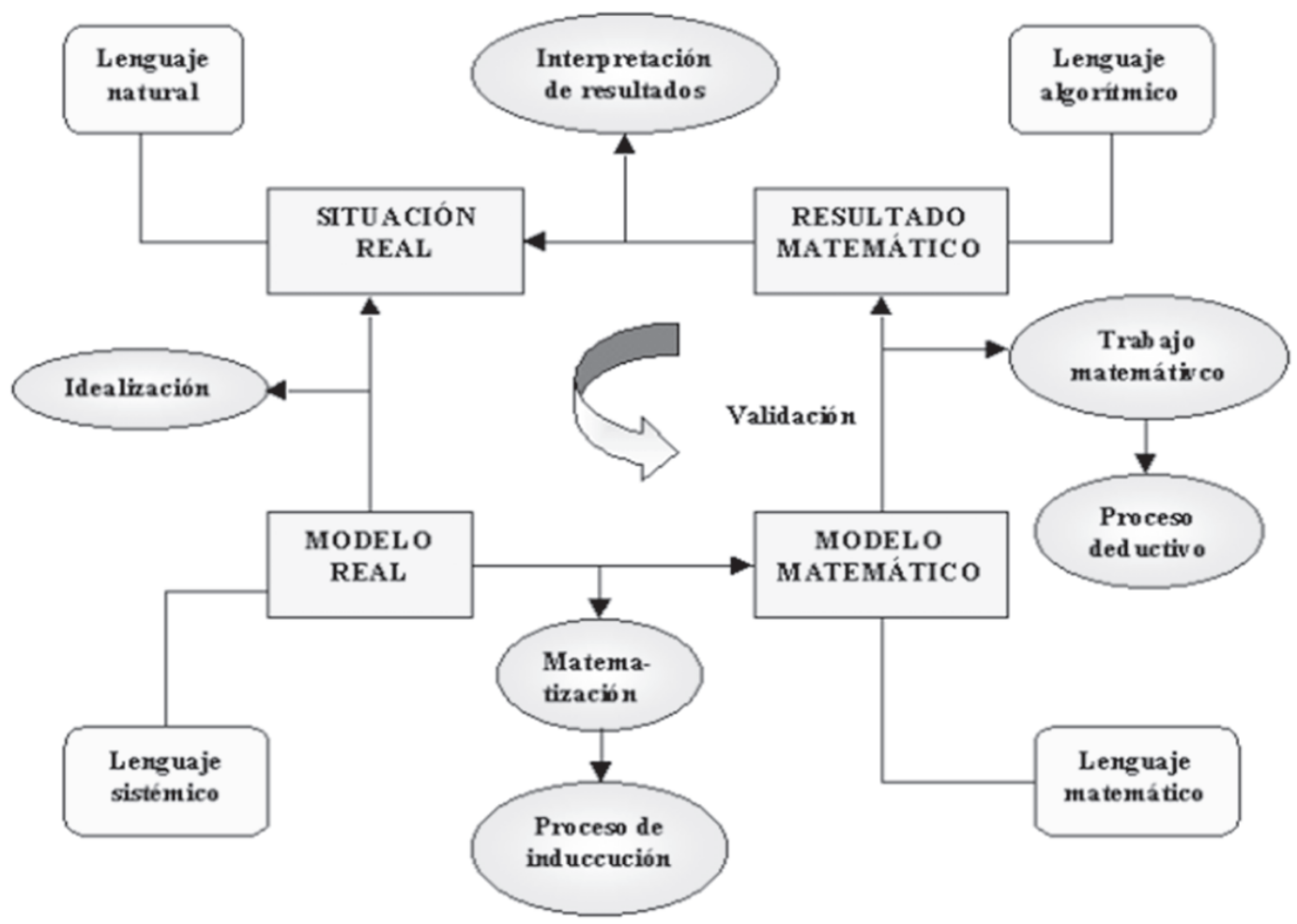

Fig. 2. Modelo de Blum.

las etapas. De otra parte el doctor Waldemar de Gregori en su obra manifiesto de la proporcionalidad con democracia directa expone lo siguiente:

"El cerebro tri uno ocupa el centro del centro por ser el núcleo de toda la creación humana, es el resumen del universo. El colorido representa tres colores básicos. El espiral tri-ramificado sobre el cerebro es la base en un simbolo tríadico de la energía, representa la tri-una proporcionalidad» [22].

Continuando con nuestra exposición es importante valorar que desde esta perspectiva tenemos tres bloques integrados e intercomunicados como puente interactor entre ellos es así como en los contextos escolares se hace necesario crear un currículo antropogógico donde se replantea el currículo y la educación reconociendo los niveles del evolución del tricerebral donde tiene mucha importancia el CCT donde se regula realmente a marcha evolutiva de mundo. Desde este punto de vista nuestra perspectiva apunta a desarrollar una estrategia didáctica centrada en el modelamiento matemático para el afianzamiento del concepto de función en los estudiantes del curso de Pre cálculo en la cual se incorporan actividades para el potenciamiento y desarrollo de los tres cerebros.

Esta propuesta se enfoca de esta manera dado que por un lado la fragmentación del conocimiento en lo referente con el concepto de función y falta de incorporación de la argumentación dentro del campo de la enseñanza de las matemáticas, dado que dentro del equipo consideramos necesario incluir el ¿Por qué de todas y cada una de las temáticas en los cursos de matemáticas?

Finalmente desde este trabajo se hace posible la incorporación de procesos futuristas que permitan abordar de manera positiva otros cursos de matemáticas y todo tipo de situaciones matemáticas dado la integralidad de la enseñanza que buscamos.

\section{Metodología}

El trabajo de investigacion se apoya en el enfoque mixto. Para el tipo de investigacion se aplicó una prueba diagnóstica que tenia como proposito ingadar sobre los diferentes conceptos 
de funcion, sus usos y aplicaciones, por otra parte se utilizó las revision de notas, talleres y pruebas para ofrecer una vision conjunta, esta constaba de una etapa posterior de analisis con los resultados encontrados. Este trabajo es descriptivo interpretativo comparativo, porque se persigue principalmente comprender la realidad estudiada desde la cual vamos a realizar una propuesta pedagógica con el propósito de generar un nueva forma de reconocer e interpretar el concepto de función, sus diferentes interpretaciones, aplicaciones y usos.

El enfoque de investigación mixto corresponde en sentido amplio la investigación mixta mezcla los enfoques cuantitativo y cualitativo [23] permiten hacer un análisis mucho más integral del fenómeno de estudio que para nuestro caso corresponde al desarrollo del concepto que tienen de función los estudiantes de un curso de pre calculo mediante una propuesta pedagógica y su evaluación, la posibilidad real se determina desde estas dos perspectivas de las variables que intervienen en este fenómeno.

Seguidamente con el desarrollo encontramos que desde este enfoque se permite diferentes formas de recolección de la información mitigando las posibilidades de errores en el desarrollo de la investigación [24]. Como segunda medida se establece [25-27] lo siguiente:

Correlación entre métodos cualitativos, cuantitativos, nuestro marco teórico con la propuesta pedagógica entorno de nuestro proyecto, mejor interpretación de los resultados del diagnóstico y las diferentes pruebas en coherencia con la propuesta pedagógico para los estudiantes a la Universidad Jorge Tadeo Lozano del curso de pensamiento matemático, desde la propuesta diseñada, nuestro abordaje del concepto de función y propuesta pedagógica se hace de manera completa desde los diferentes componentes como resultado de la propuesta, con base en los resultados del diagnóstico y los demás instrumentos, determinar las posibles contradicciones entre el marco teórico y los resultados arrojados por los instrumentos para hacer las respectivas reformulaciones, reconocer la proyección de la problemática que nuestro caso corresponde al concepto de función en el curso de pensamiento matemático.
La justificación para la aplicación del método mixto se centra en aspectos tales como la triangulación para realizar un estudio mucho más detallado de las diferentes variables que intervienen en nuestro proceso de investigación, además realizar una mejor interrelación con el marco teórico, la identificación de las debilidades y dificultades presentes en los estudiantes del curso que se está tomamos para nuestro proceso y como resultado de ello diseñar la propuesta didáctica apoyada en el modelamiento y la teoría tricebral.

El curso de Pre cálculo se ofrece para todos aquellos estudiantes que en sus programas de pregrado tiene la necesidad de abordar elementos y conceptos de pre cálculo, este curso es un prerrequisito para tomar el curso de cálculo diferencial. Se trabajó con un total de 18 estudiantes, entre ellos 10 mujeres que corresponde al 55,5\% y 8 hombres que corresponde al $44,5 \%$. Se aplicó el test del cociente tríadico a estos estudiantes y estos fueron los resultados:

Tabla 2. Resultados.

\begin{tabular}{ccc}
\hline Cerebro izquierdo & Cerebro central & Cerebro derecho \\
\hline 32,83 & 36,56 & 34,83
\end{tabular}

\section{Resultado}

Como elementos importantes para el análisis tenemos los siguientes aspectos:

\section{A. Diagnóstico. Dificultades}

Con base en el diagnóstico encontramos elementos importantes dentro del curso de precálculo estudiado y además con base en la fundamentación establecimos las categorías que mencionamos a continuación:

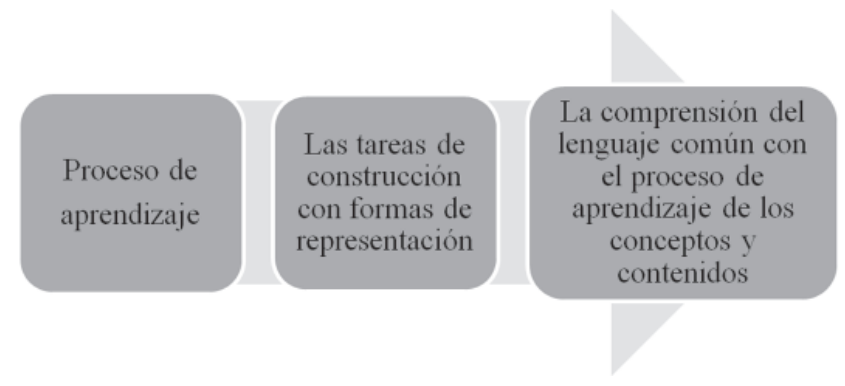

Fig. 3. Categorías. 


\section{1) Con referencia a los procesos de aprendizaje}

La distinción entre la variable y la incógnita y el poco énfasis entre la representación en términos de X y Y a diferencia de la ecuación desconociendo el concepto de función, es allí donde los estudiantes lo asocian en términos de correspondencia entre magnitudes, conjunto de números dejando sesgado y parcializado el concepto, finalmente al respecto no hay una idea clara y concreta de la relación entre variables.

\section{2) Las tareas de construcción con formas de representación}

Al manejo algebraico de la función y ecuación, no estableciendo una diferencia clara entre el manejo de función y las operaciones entre funciones

y las diferentes formas de representación de las funciones dado que es de vital importancia tener clara cuando una relación es o no una función, es allí donde nuestros estudiantes manejan el proceso de construcción de la gráfica con ayuda de la tabla sin embargo los estudiantes no reconocen como dominio y rango de las funciones en el conjunto ,no consideran las variables, no grafican y ni operan funciones de manera diferente como lo hacen con las ecuaciones dejando parcializado el concepto integral de función.

\section{3) La comprensión del lenguaje común en el proceso de aprendizaje de los contenidos y conceptos}

Los procesos algorítmicos dejan de lado todas las características de las funciones, desconocen la prueba de las líneas verticales y hay desconocimiento del metalenguaje matemático donde se usan diferentes tipos de gráficos geométricos dentro del campo de las funciones encontrándose desconexión entre el sistema lingüístico y el lenguaje matemático elementos fundamentales para la comprensión e interpretación del concepto de función.

\section{B. Nivel de competencias}

Es importante aclarar que para determinar el nivel de competencia que manejan los estudiantes, se propone una clasificación de las competencias que se deben manejar en matemáticas, esta clasificación se hace teniendo en cuenta las señaladas por PISA y las evaluadas por el icfes, con algunas pequeñas modificaciones, estas son:
Organización e Interpretación: Habilidad para interpretar y comprender el problema que se da en un contexto real, leer la información presentada en distintos formatos como tablas, gráficos, conjuntos de datos, diagramas o esquemas y extraer de ellos la información relevante.

Formulación, representación y ejecución: La capacidad para representar la situación en términos matemáticos, por medio de tablas, expresiones algebraicas, graficas o esquemas, plantear y diseñar estrategias que permitan su solución.

Argumentación y proposición: La capacidad para proponer y evaluar alternativas de solución; verificar y validar la pertinencia de dicha solución.

Estas competencias se deben evidenciar en las evaluaciones hechas durante el primer corte del semestre del curso de precalculo, razón por la cual optamos por analizar las notas de estas evaluaciones. Los resultados obtenidos se resumen en la figura 4.

Organización e Interpretación: Presenta un nivel aceptable, por cuanto no determinan las condiciones necesarias para determinar la existencia de una función, la mayoría identifica el concepto de función en gráficas, pero no en tablas o expresión algebraica.

Formulación, representación y ejecución: presenta un nivel bajo, ya que no establecen apropiadamente la relación entre las diferentes representaciones del concepto de función. No establecen con claridad cuál debe ser el modelo a utilizar para resolver los problemas de función lineal.

Argumentación y proposición: presenta un nivel bajo, pues no consiguen realmente dar solución a los problemas planteados sobre función lineal, en algunos casos se dan respuestas numéricas, pero no se contrasta con las condiciones del problema para validar dicha solución.

\section{Discusión}

Para el análisis de resultados se estableció la relación entre los tres cerebros, las competencias que se abordan en matemáticas y las dificultades que presentan los estudiantes en el estudio de las matemáticas, se ve en la tabla 3. 

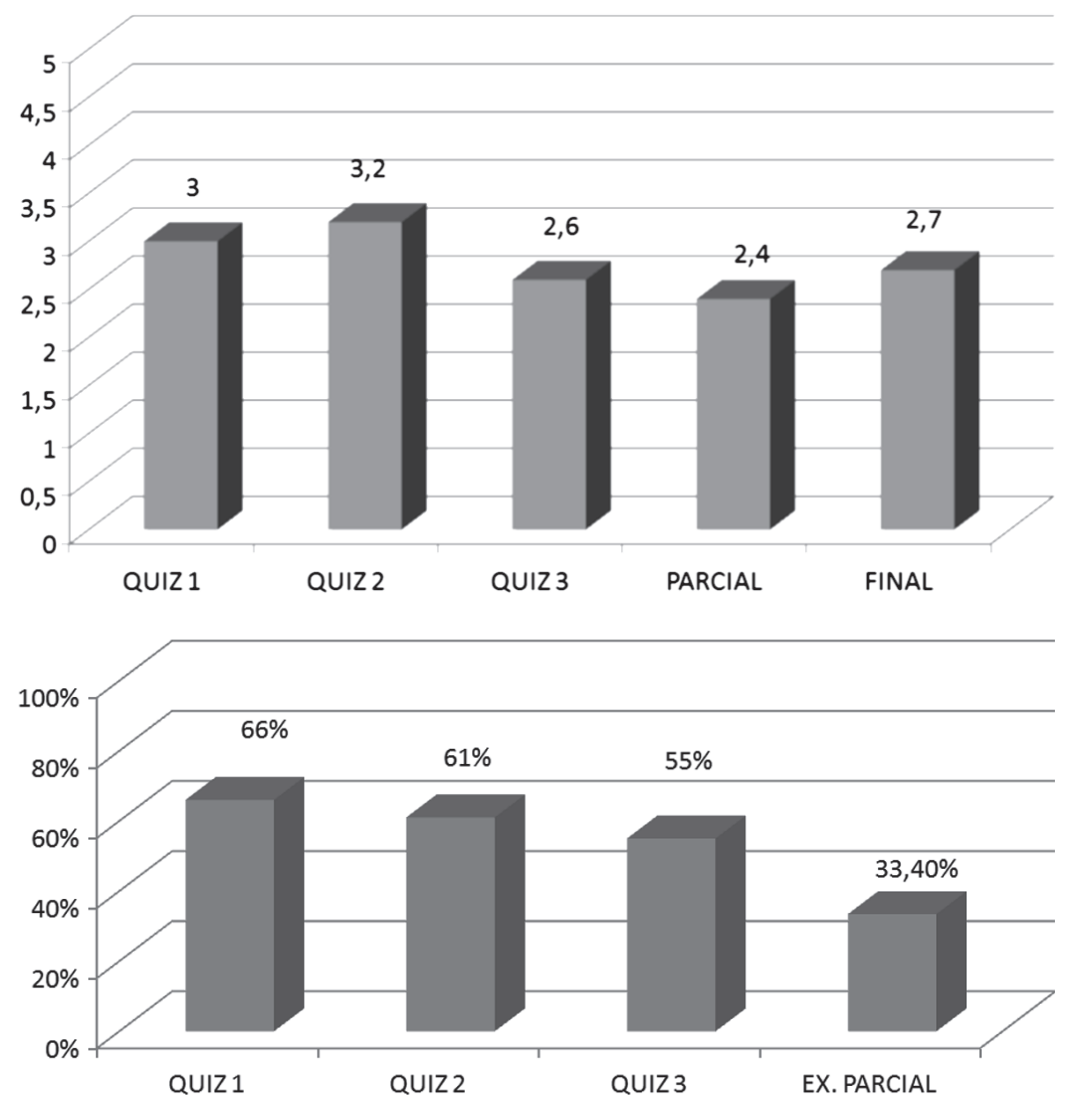

Fig. 4. Porcentaje de aprobación y promedio en cada una de las evaluaciones.

Tabla 3. Relación entre los tres cerebros.

\begin{tabular}{llll}
\hline & Cerebro lógico & Cerebro operativo & Cerebro creativo \\
\hline Competencias & Organización e Interpretación & $\begin{array}{l}\text { Formulación, representación } \\
\text { y ejecución }\end{array}$ & Argumentación y proposición. \\
\hline Dificultades & $\begin{array}{l}\text { Distinción entre los tipos } \\
\text { de variables. }\end{array}$ & $\begin{array}{l}\text { El manejo algebraico de la } \\
\text { función, sus operaciones y el } \\
\text { concepto de función. }\end{array}$ & $\begin{array}{l}\text { Los procesos algorítmicos que } \\
\text { desconocen las características de } \\
\text { funciones. }\end{array}$ \\
\hline
\end{tabular}

Cuando los estudiantes deben resolver un problema requieren del conocimiento de fórmulas, definiciones y algoritmos, por lo general estos procedimientos se han trabajado y memorizado en la clase, sin embargo, no se dedica mucho tiempo para reflexionar sobre las implicaciones de sus respuestas. En términos de competencias se podría decir que desarrollan más las competencias que con anterioridad se han relacionado con el cerebro lógico y el operativo, dejando rezagado a las correspondientes al cerebro creativo.

Es un tipo de enseñanza más adecuada porque desde la propuesta se diseñan y desarrollan acti- vidades que permiten el desarrollo y potenciación de los tres cerebros y conectar los componentes teóricos.

La enseñanza estimula el razonamiento en especial si desde las practicas pedagógicas se pueden conectar las ideas y conceptos, con lo práctico y emocional es posible la construcción de un aprendizaje significativo en el contexto del curso de pensamiento matemático.

Resulta ser un aprendizaje más integral dado que considera actividades específicas para cada uno de los cerebros como constructor de 
conocimiento desde el tricerebral y el modelamiento matemático. Es una manera de planificar y gestionar contenidos teóricos mediante actividades apoyadas en la teoría tricerebral y el modelamiento matemático.

Se propone una estrategia didáctica que se apoya en las categorías del marco teórico para una transformación en las prácticas pedagógicas en la enseñanza de las matemáticas con relación al concepto de función.

En concordancia con los trabajos de Guevara y Planchard, encontramos una marcada tendencia en los estudiantes a tratar resolver problemas usando solo algoritmos, ante esto, se deben abandonar las practicas rutinarias y convencionales para dar paso a la inclusión de la modelación, la visualización y diferentes formas de representación en la enseñanza del concepto de función [4] [5]. Adicionalmente, con referencia al problema desde el punto de vista epistemológico el concepto de función ha tomado un direccionamiento contrario dado que la última forma que precede a la enseñanza considera como herramienta de actividad matemática o extra matemática [8].

No es solamente «entrenar» a los jóvenes para utilizar la matemática en determinadas situaciones hipotéticas expuestas por el profesor, con patrones definidos y estudiados con anterioridad, para que puedan utilizar herramientas o algoritmos que se han establecido como apropiados, es decir, volverlos hábiles calculistas, la finalidad es darles la oportunidad para que puedan razonar críticamente, poder predecir y elegir de manera razonable lo mejor para el beneficio colectivo ante situaciones reales en tiempo real, enfrentándose así a innumerables variables.

Después de hacer los análisis respectivos de los resultados obtenidos, se hace necesario construir un esquema del proceso de modelación, el cual se ajusta a lo expuesto en el marco teórico, los resultados encontrados en la investigación y los objetivos propuestos, el cual servirá para implementar una propuesta de actividades basadas en la modelación que apoyen la enseñanza del concepto de función. Ver figura 5.

En este esquema se identifican dos espacios, bien diferenciados, el mundo real y el mundo matemático, pero conectados a través de tres fases, en cada una de ellas se establecen las competencias necesarias para resolver un problema, de igual forma, cada una de estas se encuadra con cada uno de los tres cerebros. Las fases son:

Situación problema, allí se pretende lograr la interpretación y comprensión del problema que se da en un contexto real. (Cerebro lógico).

Modelo matemático, en esta etapa se logra trasladar al mundo matemático por medio de fórmulas, ecuaciones y cualquier otro objeto matemático la representación de la situación. También se efectúan los cálculos y operaciones necesarias para encontrar un resultado matemático. (Cerebro operativo).

Posible solución, después de hacer operaciones y cálculos en el mundo matemático, se transfiere

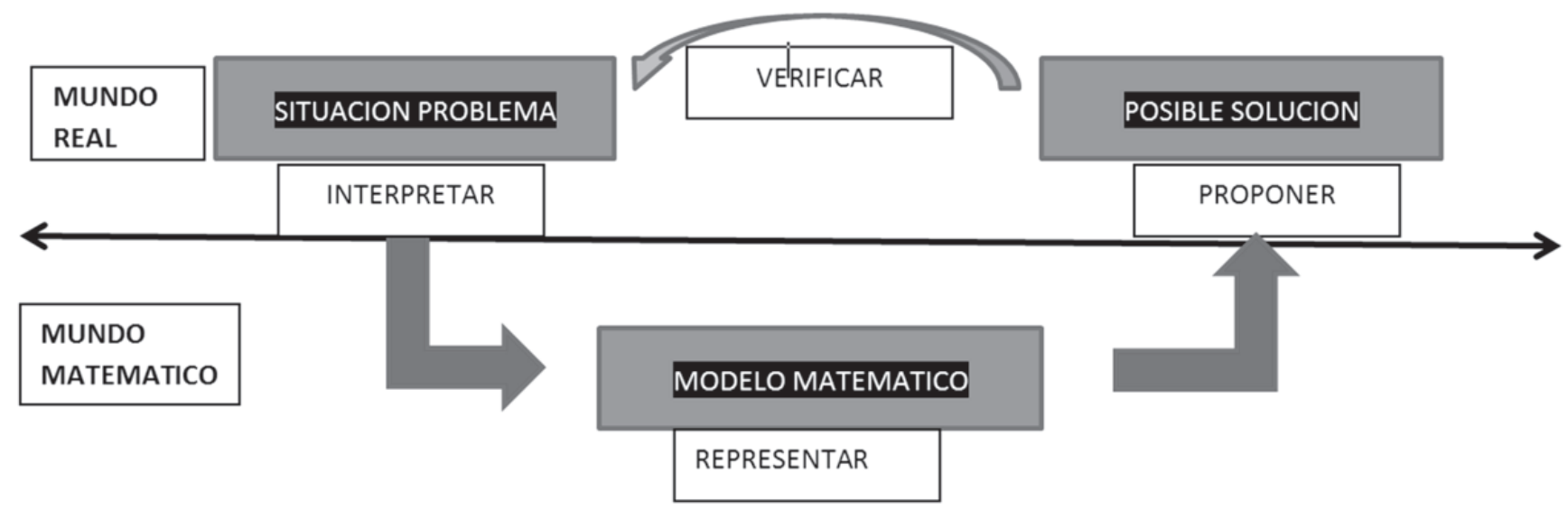

Fig. 5. Esquema problema- modelo matemático - Posible solución. 
la respuesta que se obtuvo, es decir volver al mundo real y tomar alguna decisión con respecto a la solución encontrada, se proponen alternativas de solución. (Cerebro creativo).

\section{REFERENCIAS}

[1] M. Spivak, Calculus Cálculo infinitesimal, Segunda. Universidad de Brandeis: Reverté, S.A, 1992.

[2] J. Stewart, Cálculo de una variable. Conceptos y contextos, Sexta. Cengage Learning, 1999.

[3] W. Blum, M. Niss, P. Galbraith and H. Henn, Modelling and aplications in mathematics education. The 14th ICMI Study: New York: Springer, 2007.

[4] C. Guevara, «Propuesta didáctica para lograr aprendizaje significativo del concepto de función mediante la modelación y la simulación,» Universidad Nacional de Colombia Sede Medellín, Medellín, 2011.

[5] E. Planchard, «Clasificación de los modelos pedagógicos,» 2012.

[6] F. Córdoba, «La modelación en matemática educativa: una práctica para el trabajo de aula en ingeneniería,» Instituto Politécnico Nacional, México, 2011.

[7] W. Ugalde, «Funciones: desarrollo histórico del concepto y actividades de enseñanza aprendizaje,» Revista digital - Matemática, Educación e Internet, vol. 14, No. 1, p. 48, 2014.

[8] L. Ruiz, «La noción de función: análisis epistemológico y didáctico.» Universidad de Jaén. Servicio de publicaciones, Jaén, 1998.

[9] C. Cuevas and J. Díaz, «La historia de la matemática un factor imprescindible en la elaboración de una propuesta didáctica. El caso del concepto de función.» Departamento de Matemáticas, Universidad de Sonora.

[10] M. Artigue, R. Douady, L. Moreno, Ingeniería Didáctica en educación matemática. Un esquema para la investigación y la innovación en la enseñanza y el aprendizaje de las matemáticas, Universidad de los Andes. Bogotá: Grupo Editorial Iberoamérica, 1995.

[11] E. Ruhana, M. Bruekheimer, «Univalente: A Critical on non-critical characteristics of functions? For the learning in mathematics.» 1998.

[12] G. Leinhardt, O. Zaslavsky, M. Stein, «Functions, Graphs and Graphing: Task, Learning and Teaching.» Review of Educational Research, 1990.

[13] D. Tall, S. Vinner, Concept image and concept definiton in mathematics, with particular reference to limits and continuity., Educational Studies in Mathematics. 1981.

[14] C. Azcárate, «Sistemas de Representación.,» UNO. Revista de Didáctica de las Matemáticas, 1990.

[15] C. Janvier, «The interpretation of complex cartesian graphs representing situations,» University of Nottingham, 1978.

[16] R. Lesh, T. Post, M. Behr, Representation and translations among representations in mathematics learning and problem solving. N.J: Lawrence ER, 1987.

[17] C. Monereo, M. Castelló, «Las estrategias de aprendizaje. Cómo incorporarlas a la práctica educativa.» Edebé, 1997.

[18] Ministerio de Educación Nacional, «Competencia,» MEN. Colombia, 2006.

[19] Departamento de educación, universidades e investigación., «Las competencias básicas en el Sistema Educativo de la C.A.P.V. .» Gobierno vasco, 2009.

[20] Instituto Nacional de Evaluación Educativa, «5 Claves para entender la competencia matemática en \#PISA,» Educalab.

[21] L. Gutiérrez, «Gutiérrez, Luz (2008) Competencia básicas en el área de matemáticas.» Consejería de educación del gobierno de Cantabria, 2008.

[22] W. Gregori, «Manifiesto de la Proporcionalidad.» 2008.

[23] B. Johnson, A. Onwuegbuzie, «Methods Research: A Research Paradigm Whose Time Has Come [Los métodos de investigación mixtos: un paradigma de investigación cuyo tiempo ha llegado],» Educational Researcher, vol.33, no. 7, pp. 14-26, Oct2004.

[24] R. Sampieri, C. Collado, P. Lucio., Metodología de la investigación. Mexico, D.F: McGraw Hill Interamericana, 2003.

[25] J. C. Greene, Mixed methods in social inquiry. San Francisco, CA, EE. UU: Jossey-Bass, 2008.

[26] A. Tashakkori, C. Teddlie, Introduction to mixed method and mixed model studies in the social and behavioral sciences. The mixed methods reader, In V. L. Plano y J. W. Creswell (Eds.). Thousand Oaks, CA, EE. UU: Sage, 2008.

[27] R. Hernández Sampieri y C. P. Mendoza, El matrimonio cuantitativo cualitativo: el paradigma mixto., Congreso efectuado por el Instituto Mexicano de Sexología. Villahermosa, Tabasco, México.: Universidad Juárez Autónoma de Tabasco, 2008.

Rev. Ingeniería, Matemáticas y Ciencias de la Información Vol. 3 / Núm. 5 / enero-junio de 2016; pág. 37-50 